\title{
Trombosis de seno lateral como complicación de otitis media aguda en niños. Reporte de un caso y revisión de la literatura
}

\section{Lateral sinus thrombosis as a complication of acute otitis media in children. A case report and literature review}

\author{
Catalina Gutiérrez C', Loreto Carrasco M², Maritza Rahal E².
}

\begin{abstract}
RESUMEN
La otitis media aguda es frecuente en niños y generalmente de evolución benigna. Durante las últimas décadas, con el uso de antibióticos y tratamientos quirúrgicos asociados, la incidencia de las complicaciones de la otitis media aguda ha disminuido hasta 10 veces junto con la mortalidad de éstas (menor al 10\%). Las complicaciones se pueden clasificar según su localización en intratemporales o intracraneales. Dentro de las complicaciones intracraneales más frecuentes, se encuentra la trombosis del seno lateral.

En el presente estudio se reporta el caso de un preescolar de 5 años que fue derivado al Servicio de Otorrinolaringología del Hospital Barros Luco Trudeau por otitis media aguda complicada con mastoiditis y absceso subperióstico que fue drenado junto con una mastoidectomía. Cuatro días posterior a la cirugía se agrega cefalea, vómitos no explosivos y edema de papila por lo que se realiza una resonancia nuclear magnética en fase venosa que muestra trombosis del seno lateral. Se realiza una revisión de la literatura sobre el manejo de la trombosis del seno lateral y, basado en la evidencia existente, se propone un algoritmo de manejo para esta patología ya que aún no existe un consenso para su tratamiento.
\end{abstract}

Palabras claves: Otitis media aguda, complicaciones, trombosis seno lateral, tromboflebitis.

\begin{abstract}
Acute otitis media is a common disease in children and generally has a benign course. During recent decades, the use of antibiotics and associated surgical treatments has decreased the incidence of the complications of acute otitis media in 10 times, as well as the mortality which currently is less than 10\%. According to their location, the complications can be classified in intratemporal or intracranial. Lateral sinus thrombosis is one of the most common intracranial complications of acute otitis media.
\end{abstract}

Médico Cirujano. Magíster en Epidemiología, Universidad de Los Andes.

2 Médico. Servicio Otorrinolaringología, Hospital Barros Luco Trudeau. 
The present study reports the case of a 5 year old child who was referred to our Department of Otolaryngology for acute otitis media complicated with mastoiditis and subperiosteal abscess. He underwent a mastoidectomy and the abscess was drained. Four days after surgery the child presented headache, nonexplosive vomiting and papilledema. Lateral sinus thrombosis was identified by magnetic resonance venography. We present a literature review on the management of this pathology, and, based on existing evidence, we propose an algorithm for the management of this condition because there is still no consensus for its treatment.

Key words: Acute otitis media, complications, lateral sinus thrombosis, thrombophlebitis.

\section{INTRODUCCIÓN}

La otitis media aguda (OMA) es una enfermedad frecuente en la infancia: entre los 6 y 11 meses de vida, el $19 \%$ al $62 \%$ de los lactantes ha tenido al menos un episodio de OMA; a los 3 años, entre el $50 \%$ y $85 \%$ ha presentado un cuadro; y a los 5 años de edad, entre el $60 \%$ y $90 \%$ ha tenido un episodio. La mayoría de las veces éste es un proceso benigno. Alrededor de $80 \%$ de los niños con OMA presentarían una resolución clínica entre los 3 y los 7 días tras su diagnóstico sin el uso de antibióticos y con un bajo riesgo de complicaciones.

Las complicaciones de la otitis media (OM) se definen como la extensión de la infección más allá de los confines de los espacios neumatizados del hueso temporal y su mucosa. Según su localización, éstas se pueden clasificar en intratemporales 0 intracraneales. Durante las últimas décadas, con el uso de antibióticos y los tratamientos quirúrgicos asociados la incidencia de las complicaciones ha disminuido hasta 10 veces junto con la mortalidad de éstas (de $40 \%$ a menos del $10 \%$ ). Dentro de las complicaciones intracraneales más frecuentes, se encuentra la trombosis del seno lateral (TSL) que da cuenta de entre el $17 \%$ y $19 \%$ de las complicaciones intracraneales en niños. Sin embargo, el tratamiento de la trombosis del seno lateral, aún no tiene un consenso.

En el presente estudio se reporta el caso de un niño con TSL y luego una revisión de la literatura sobre el manejo de la TSL.

\section{CASO CLÍNICO}

Paciente preescolar de 5 años de edad, de sexo masculino, sin antecedentes mórbidos de importancia, con cuadro de virosis respiratoria alta de 10 días de evolución, agregándose posteriormente otalgia derecha y fiebre hasta $41^{\circ} \mathrm{C}$. Consultó en atención primaria donde se diagnosticó OMA derecha, iniciándose tratamiento con gotas óticas y amoxicilina en dosis de $60 \mathrm{mg} / \mathrm{kg} / \mathrm{día}$. A pesar del tratamiento, al tercer día evolucionó con aumento de volumen retroauricular derecho y vómitos por lo que fue derivado al Servicio de Otorrinolaringología del Hospital Barros Luco en octubre del año 2009. A su ingreso se encontraba decaído, con aumento de volumen retroauricular derecho fluctuante, eritematoso y sensible a la palpación. A la otomicroscopía se encontró pus en el conducto auditivo externo y una perforación central pequeña con otorrea pulsátil. Se diagnosticó OMA complicada con otomastoiditis derecha y se decidió hospitalizar para estudio y tratamiento antibiótico endovenoso con ceftriaxona, cloxacilina y penicilina.

Dentro de los exámenes de ingreso destacaban una proteína C reactiva (PCR) de 190,8 mg/dl y una leucocitosis de 20.480 por $\mathrm{mm}^{3}$. En la tomografía computarizada (TC) de peñasco, cerebro y fosa posterior del 20 de octubre, se observaba un velamiento mastoideo, alistéresis de las celdillas y un absceso subperióstico mastoideo derecho (Figura 1). El día 22 de octubre se realizó el drenaje del absceso subperióstico vía retroauricular y mastoidectomía, tomándose muestras para cultivos, las que fueron informadas como negativas. Además, se tomaron muestras para estudio anatomopatológico, que se informaron como tejido inflamatorio.

Cuatro días después se agregó cefalea y vómitos no explosivos. Fue evaluado por oftalmólogo, quien constató edema de papila bilateral y restric- 


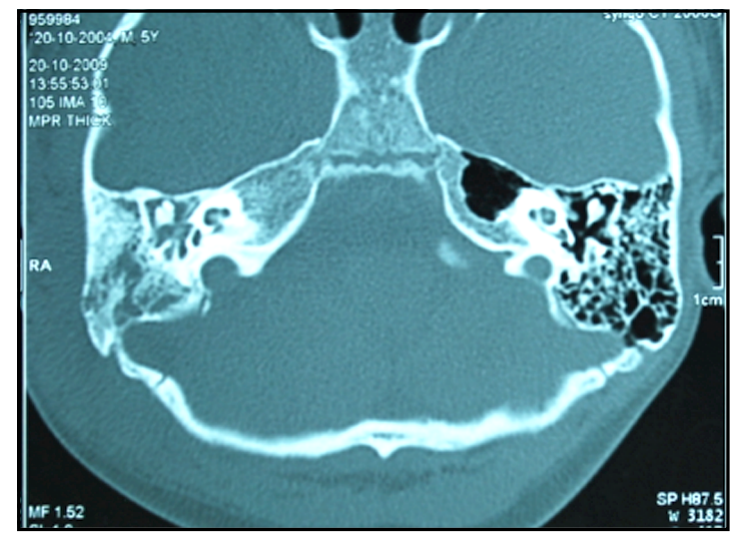

Figura 1. TC de oído, corte axial, donde se observa ocupación de oído medio derecho y alistéresis de las celdillas mastoideas ipsilaterales, asociado a lesión del hueso cortical en esa región.

ción mínima del campo visual. Se realizó una resonancia nuclear magnética (RNM) en fase venosa, observándose una pequeña colección extradural de fosa posterior derecha y un déficit de contrastación del seno venoso lateral derecho con refuerzo dural, que se interpretó como una tromboflebitis (Figura 2). Por esto, se decidió iniciar tratamiento anticoagulante con Fragmin 2500 UI subcutáneo cada 12 hrs.

Tras la evaluación por neurología, se decidió manejo y monitorización en Unidad de Cuidados Intermedios Pediátrico. El paciente evolucionó favora- blemente, afebril y con disminución progresiva de la cefalea, náuseas y vómitos. Hubo regresión del compromiso papilar y normalización de los parámetros inflamatorios. Completó tratamiento antibiótico por 5 semanas y anticoagulante por 6 meses en total.

Se realizaron RNM en fase venosa de control al mes, a los dos meses y al sexto mes del diagnóstico, observándose recanalización parcial del seno sigmoideo derecho. El paciente evolucionó asintomático.

Los diagnósticos de alta fueron: OMA derecha complicada con otomastoiditis aguda derecha, abs-

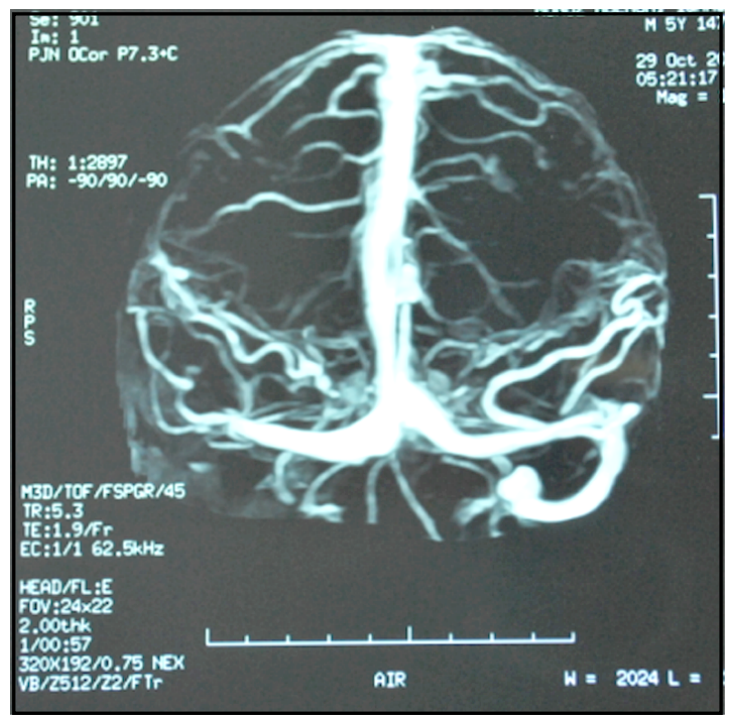

Figura 2. RNM cerebro, fase venosa. Se observa la trombosis del seno lateral derecho. 
ceso subperióstico mastoideo derecho drenado, absceso extradural y tromboflebitis del seno sigmoideo derecho parcialmente recanalizado.

\section{DISCUSIÓN}

Las complicaciones de las OM se pueden clasificar según su mecanismo patogénico (infeccioso o no infeccioso) o según su localización. Esta última, la más utilizada, las divide en intratemporales (extracraneales u otológicas) e intracraneales (0 endocraneales). Dentro de las complicaciones intratemporales se encuentran la mastoiditis, laberintitis, parálisis facial y la petrositis (algunos la consideran intracraneal). Por su parte, las complicaciones intracraneales incluyen la meningitis, abscesos cerebrales y TSL (específicamente seno sigmoideo), entre otras. Las complicaciones más frecuentes son las intratemporales, específicamente la mastoiditis, y dentro de las intracraneales la meningitis es la de mayor incidencia.

La TSL como complicación de la OM es un cuadro poco frecuente. Kangsanarak en un estudio retrospectivo de 12 años en una región de Tailandia encontró 6,5 casos de TSL por 10.000 casos de $\mathrm{OM}^{13}$. Goldenberg en un estudio en Estados Unidos reportó una incidencia de 0,625 casos por Departamento de Otorrinolaringología en 5 años. La TSL secundaria a OMA se presenta principalmente en niños. Cuando se presenta en adultos, la mayoría de las veces está asociada a otitis media crónica, ya sea simple o colesteatomatosa. El cuadro clínico que debe hacer sospechar una TSL es la otalgia asociada a cefalea, náuseas y vómitos. Durante los últimos años ya no se observa la fiebre en agujas típica de este cuadro, sino más bien una fiebre baja 0 incluso puede no estar presente en algunos pacientes. Esto se explicaría por el amplio uso de antibióticos en los cuadros de OMA. Dentro de los signos neurológicos más frecuentes se encuentran la parálisis del nervio abducens (los pacientes pueden referir diplopía) y parálisis facial intrapetrosaADDIN EN.CITE.DATA ${ }^{9,17,20-29}$. La trombosis venosa puede impedir el drenaje del líquido cerebroespinal (LCE), aumentando la presión intracraneana y produciendo así un hidrocéfalo ótico con papiledema y aumento de la presión de salida del LCE a la punción lumbar. Otros signos menos frecuentes de TSL son edema retroauricular, otorrea, debilidad y rigidez de nuca.

Para confirmar el diagnóstico se utiliza la TC y la RNM. En la TC sin contraste la hiperdensidad del seno sugiere una TSL. En la TC con contraste se observa un defecto de llenado del seno y ocasionalmente un reforzamiento de la pared que rodea al seno que contiene el trombo (signo delta). La TC sólo diagnostica el $84 \%$ de las TSL detectadas por RNM. La RNM en fase venosa tiene el mejor rendimiento por lo que es el examen de elección para realizar el diagnóstico.

Con respecto a la microbiología, dado el uso previo de antibióticos, los cultivos son típicamente negativos en la TSL. Sin embargo, cuando se ha logrado aislar microorganismos se han encontrado principalmente Streptoccoccus, gram negativos, Staphilococcus aureus y anaerobios, los que son generalmente multirresistentes.

Respecto al tratamiento de la TSL no existen guías clínicas estandarizadas dado que los estudios publicados son de series pequeñas. Entre los tratamientos más utilizados se encuentran:

Antibioterapia: Se deben elegir antibióticos de amplio espectro y que atraviesen la barrera hematoencefálica (BHE). Las cefalosporinas de tercera generación por vía endovenosa son las más frecuentemente utilizadas. Se puede asociar vancomicina para cubrir estreptococo resistente y para cubrir anaerobios de preferencia metronidazol en vez de clindamicina ya que esta última no atraviesa la BHE. Si se logra aislar un microorganismo se inicia tratamiento específico según el antibiograma. La duración del tratamiento antibiótico varía entre 3 a 6 semanas. Los pacientes pueden completar el tratamiento endovenoso en forma ambulatoria o cambiar a tratamiento oral (en la mayoría de los estudios revisados utilizan cefadroxilo, amoxicilina con ácido clavulánico y/o metronidazol).

Tratamiento quirúrgico: Tiene el objetivo de remover el foco de infección y asegurar la ventilación del oído medio y mastoides. La cirugía a realizar varía desde la inserción de tubos de ventilación a cirugías más radicales en caso de otra complicación intracerebral asociada. En la mayoría de los casos se realiza una mastoidectomía y aspirado del seno con aguja que 
tiene como finalidad evaluar la presencia de pus 0 ausencia de retorno sanguíneo, indicadores para abrir el seno venoso. Procedimientos menos frecuentes son la embolectomía y resección del seno sigmoides. Actualmente, la ligadura de la vena yugular interna no se recomienda. En algunos casos publicados no se realizó cirugía sino que se optó por tratamiento médico con evolución favorable de los pacientes.

Anticoagulación: Es el punto de mayor controversia dentro del tratamiento de la TSL. Primero, cabe preguntarse si existe una tendencia protrombótica en los niños que presentan TSL como complicación de una OMA. Se encontraron en total 21 pacientes con estudio de factores protrombóticos. De ellos, 5 $(23,8 \%)$ presentaron alteraciones de tipo protrombótico.

Las recomendaciones para el uso de tratamiento anticoagulante (TACO) en las trombosis venosas vienen de estudios de neurología y de hematología en los cuales se excluyen los trombos de etiología infecciosa y además reportan distintas tasas de mortalidad que las TSL secundarias a OM, lo que sugiere una fisiopatología diferente detrás de ambos tipos de cuadros. Bradley hace recomendaciones más específicas para el uso de TACO en pacientes con TSL. Sugiere no usar TACO en pacientes con trombosis confinada al seno sigmoides y recomienda imagenología seriada y anticoagular sólo en caso de evidencia de progresión del trombo, extensión del trombo a otros sitios (vena yugular interna, seno transverso y seno cavernoso), cambios neurológicos, fiebre persistente o eventos embólicos.

La principal complicación asociada al uso de TACO que se describe en la literatura es la hemorragia. En cuanto a la evolución de la trombosis, a pesar del tratamiento anticoagulante, en el seguimiento de estos pacientes se encontró que algunos no estaban recanalizados. Por otra parte, se ha visto recanalización de los senos venosos aún sin el uso de TACO, por lo que según este criterio, la anticoagulación podría no ser siempre necesaria. De un total de 73 casos revisados, 33 pacientes fueron anticoagulados y 40 no. Los resultados son similares entre ambos grupos. El rol de seguir a los pacientes con imágenes para evaluar si hay 0 no recanalización es dudoso ya que no hay una aparente correlación con complicaciones a largo plazo.

Las contraindicaciones generales de uso de TACO son hemorragia intracraneana, cirugía inminente 0 coagulopatía. Sin embargo, una cirugía inminente pudiese ser una contraindicación relativa, ya que existen fármacos que revierten el efecto anticoagulante.

Llama la atención que la mortalidad por TSL descrita en la literatura menor al 10\%, es casi cero en los estudios encontrados. Los únicos casos de mortalidad por TSL encontrados son del estudio de Samuel en Sudáfrica, en que reporta 45 casos de TSL entre los años 1978 y 1984, la mayoría de los cuales están asociados a otras complicaciones intracraneanas. Dentro de este grupo se describen 4 fallecidos (8,8\% de mortalidad).

\section{CONCLUSIÓN}

El manejo de los pacientes con complicaciones intracraneales secundarias a patología ótica resulta desafiante. A continuación se presenta un algoritmo de manejo de pacientes con TSL propuesto por Christensen (Figura 3).

Frente a un paciente con el diagnóstico de OMA debemos sospechar una TSL si presenta otalgia asociado a cefalea, náuseas y vómitos; 0 si presenta una otoscopía anormal asociada a paresia o parálisis del sexto par o si la TC presenta signos sugerentes. El manejo de la TSL que proponemos es similar al de Christensen, es decir, iniciar antibióticos de amplio espectro y realizar una RNM en fase venosa. Si ésta fuese negativa para TSL, sugerimos mantener el tratamiento antibiótico y descartar otras complicaciones de OM. Si el examen confirma el diagnóstico de TSL, se debe evaluar el compromiso sistémico del paciente. Si es leve, se puede optar por un manejo médico (antibióticos) y si se encuentra séptico, agregar tratamiento quirúrgico, que a diferencia de Christensen, sugerimos una mastoidectomía con exploración del seno lateral. Por último, dado que el uso de TACO es controversial, proponemos evaluar caso a caso, indicando su uso especialmente si el estudio de factores protrombóticos resulta positivo. 


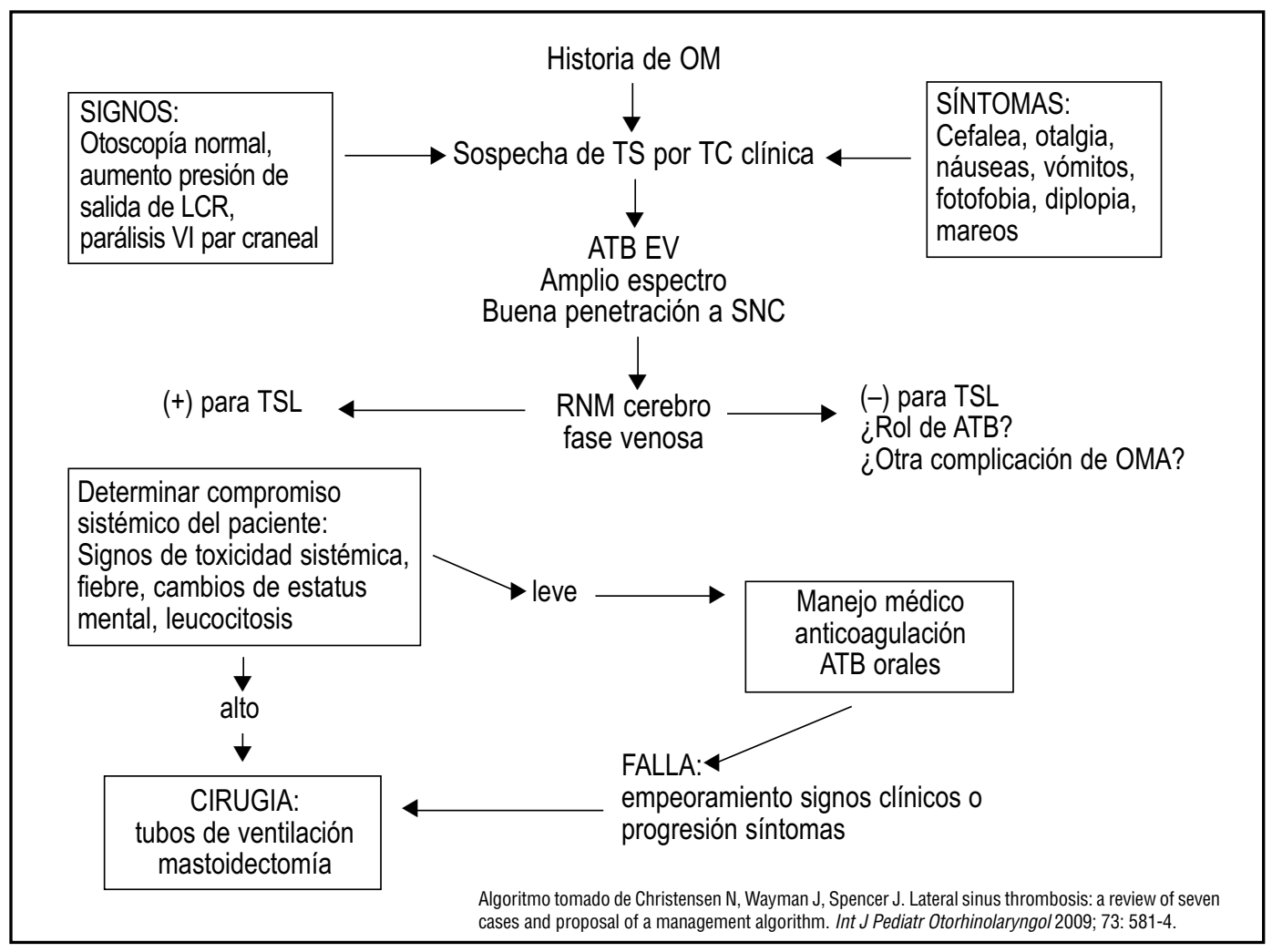

Figura 3. Algoritmo de manejo de pacientes con TSL

\section{BIBLIOGRAFÍA}

1. Casselbrant ML, Mandel E. Epidemiology. In: Evidence-Based Otitis Media. Rosenfeld R, Bluestone CD, editors. St. Louis, MO: BC Decker; 1999; 117-36.

2. Takata GS, Chan LS, Shekelle P. Evidence assessment of management of acute otitis media: I. The role of antibiotics in treatment of uncomplicated acute otitis media. Pediatrics 2001; 108: 239-47.

3. RosenfeLd R. Natural history of untreated otitis media. In: Evidence-Based Otitis Media. Rosenfeld R, Bluestone CD, editors. St. Louis, MO: BC Decker; 2001; 157-77.

4. NeELy J. Complications of temporal bone infections. In: Otolaryngology-head and neck surgery. Cummings C, Fredrickson J, Harker L, editors. St. Louis, MO: Mosby; 1993; 284064.
5. Teichgraeber JF, Per-Lee JH, Turner JS, JR. Lateral sinus thrombosis: a modern perspective. Laryngoscope 1982; 92: 744-51.

6. GLasscock MEI, Gulya AJ. Surgery of the ear. In $5^{\text {th }}$ ed. Ontario, Canada: BC Decker Inc; 2003; 443-61.

7. Wetmore RF. Complications of otitis media. Pediatr Ann 2000; 29: 637-46.

8. Ool EH, Hilton M, Hunter G. Management of lateral sinus thrombosis: update and literature review. J Laryngol Otol 2003; 117: 932-9.

9. Manolidis S, Kutz JW, JR. Diagnosis and management of lateral sinus thrombosis. Otol Neurotol 2005; 26: 1045-51.

10. Samuel J, Fernandes CM. Lateral sinus thrombosis (a review of 45 cases). J Laryngol Otol 1987; 101: 1227-9.

11. Mathews TJ. Lateral sinus pathology (22 cases managed at Groote Schuur Hospital). J Laryngol Otol 1988; 102: 118-20. 
12. deVeber G, Andrew M, Adams C, et al. Cerebral sinovenous thrombosis in children. $N$ Engl J Med 2001; 345: 417-23.

13. Kangsanarak J, Navacharoen N, Fooanant S, et al. Intracranial complications of suppurative otitis media: 13 years' experience. Am J Otol 1995; 16: 104-9.

14. Hafidh MA, Keogh I, Walsh RM, et al. Otogenic intracranial complications. a 7-year retrospective review. Am J Otolaryngol 2006; 27: 390-5.

15. Kangsanarak J, Fooanant S, Ruckphaopunt K, et al. Extracranial and intracranial complications of suppurative otitis media. Report of 102 cases. $J$ Laryngol Otol 1993; 107: 999-1004.

16. Goldenberg RA. Lateral sinus thrombosis. Medical or surgical treatment? Arch Otolaryngol 1985; 111: 56-8.

17. Wanna GB, Dharamsi LM, Moss JR, et al. Contemporary management of intracranial complications of otitis media. Otol Neurotol 2010; 31: 111-7.

18. Ruiz Sastre A, Ballester Belda Je, Ortega Gonzalez E, ET AL. [Otitis media and bacteremia. Thrombosis of the lateral sinus secondary to acute otitis media]. Rev Clin Esp 2001; 201: 409-10.

19. Sitton MS, Chun R. Pediatric otogenic lateral sinus thrombosis: role of anticoagulation and surgery. Int J Pediatr Otorhinolaryngol 2012; 76: 428-32.

20. Tov EE, Leiberman A, Shelef I, et al. Conservative nonsurgical treatment of a child with otogenic lateral sinus thrombosis. Am J Otolaryngol 2008; 29: 138-41.

21. Bales CB, Sobol S, Wetmore R, et al. Lateral sinus thrombosis as a complication of otitis media: 10 year experience at the children's hospital of Philadelphia. Pediatrics 2009; 123: 709-13.

22. García RD, Baker AS, Cunningham MJ, et al. Lateral sinus thrombosis associated with otitis media and mastoiditis in children. Pediatr Infect Dis $J$ 1995; 14: 617-23.

23. Shah UK, JubeliRer TF, FISH JD, et al. A caution regarding the use of low-molecular weight heparin in pediatric otogenic lateral sinus thrombosis. Int J Pediatr Otorhinolaryngol 2007; 71: 347-51.

24. Long YT, Mahmud R, Sani A, et al. Complications of otitis media requiring surgical intervention. Asian J Surg 2002; 25: 170-4.
25. Spandow 0, Gothefors L, Fagerlund M, et al. Lateral sinus thrombosis after untreated otitis media. A clinical problem-again? Eur Arch Otorhinolaryngol 2000; 257: 1-5.

26. Christensen N, Wayman J, Spencer J. Lateral sinus thrombosis: a review of seven cases and proposal of a management algorithm. Int $J$ Pediatr Otorhinolaryngol 2009; 73: 581-4.

27. Bradley DT, HashisakI GT, Mason JC. Otogenic sigmoid sinus thrombosis: what is the role of anticoagulation? Laryngoscope 2002; 112: 1726-9.

28. Nurliza I, Saim L. Lateral sinus thrombosis. Med J Malaysia 2007; 62: 245-6.

29. García de Hombre A, Santana P, Vera A. Trombosis de los senos venosos, complicación tardía post mastoiditis en una preescolar. Rev Chil Pediatr 2005; 76: 384-88.

30. Goldberg Al, Rosenbaum ae, Wang h, et al. Computed tomography of dural sinus thrombosis. J Comput Assist Tomogr 1986; 10: 16-20.

31. HARRIS TM, Smith RR, Косн KJ. Gadolinium-DTPA enhanced MR imaging of septic dural sinus thrombosis. J Comput Assist Tomogr 1989; 13: 682-4.

32. Croche Santander B, Porras Gonzalez A, Madrid CASTILLO MD, ET AL. [Unusually high frequency of complications in acute otitis media]. An Pediatr (Barc) 2009; 70: 168-72.

33. Bianchini C, Aimoni C, Ceruti S, et al. Lateral sinus thrombosis as a complication of acute mastoiditis. Acta Otorhinolaryngol Ita/2008; 28 : 30-3.

34. KelLy KE, JackLeR RK, Dillon WP. Diagnosis of septic sigmoid sinus thrombosis with magnetic resonance imaging. Otolaryngol Head Neck Surg 1991; 105: 617-24.

35. Iseri M, Aydin 0, Ustundag E, et al. Management of lateral sinus thrombosis in chronic otitis media. Otol Neurotol 2006; 27: 1098-103.

36. Kaplan DM, Kraus M, Puterman M, et al. Otogenic lateral sinus thrombosis in children. Int J Pediatr Otorhinolaryngol 1999; 49: 177-83.

37. Oestreicher-Kedem Y, Raveh E, KornReich L, et al. Prothrombotic factors in children with otitis media and sinus thrombosis. Laryngoscope 2004; 114: 90-5. 
38. Bielecki I, Cofala M, MierzWinski J. Otogenic lateral sinus thrombosis in children. Otol Neurotol 2011; 32: 1111-5.

39. Oyarzabal MF, Patel KS, Tolley NS. Bilateral acute mastoiditis complicated by lateral sinus thrombosis. J Laryngol Otol 1992; 106: 535-7.

40. Wong I, KozAK FK, PoskitT K, et al. Pediatric lateral sinus thrombosis: retrospective case series and literature review. J Otolaryngo/2005; 34: 79-85.

41. Kuczkowski J, Dubaniewicz-Wybieralska M, Przewozny T, et al. Otitic hydrocephalus associated with lateral sinus thrombosis and acute mastoiditis in children. Int $J$ Pediatr Otorhinolaryngol 2006; 70: 1817-23.

42. Einhaupl KM, Villringer A, Meister W, et al. Heparin treatment in sinus venous thrombosis.
Lancet 1991; 338: 597-600.

43. deVeber G, Chan A, Monagle P, et al. Anticoagulation therapy in pediatric patients with sinovenous thrombosis: a cohort study. Arch Neurol 1998; 55: 1533-7.

44. Ameri A, Bousser MG. Cerebral venous thrombosis. Neurol Clin 1992; 10: 87-111.

45. Bousser MG. Cerebral venous thrombosis: diagnosis and management. J Neuro/2000; 247: 252-8.

46. Neilan RE, Isaacson B, Kutz JW, JR., et al. Pediatric otogenic lateral sinus thrombosis recanalization. Int J Pediatr Otorhinolaryngol 2011; 75: 850-3.

47. AgarWal $A$, Lowry $P$, IsaAcson G. Natural history of sigmoid sinus thrombosis. Ann Otol Rhinol Laryngol 2003; 112: 191-4. 